\title{
microRNA-155 regulates cell proliferation and invasion by targeting FOXO3a in glioma
}

\author{
NAN LING ${ }^{1 *}$, JUNYI GU ${ }^{1 *}$, ZHE LEI ${ }^{2,3}$, MENGMENG LI' ${ }^{2,3}$, JUN ZHAO $^{3,4}$, \\ HONG-TAO ZHANG ${ }^{2,3}$ and XIANGDONG LI ${ }^{1,3}$
}

\author{
${ }^{1}$ Department of Neurosurgery, The First Affiliated Hospital of Soochow University, Suzhou 215006; \\ ${ }^{2}$ Soochow University Laboratory of Cancer Molecular Genetics, Medical College of Soochow University, \\ Suzhou 215123; ${ }^{3}$ Suzhou Key Laboratory for Molecular Cancer Genetics, Suzhou 215123; \\ ${ }^{4}$ Department of Surgery, The First Affiliated Hospital, Soochow University, Suzhou 215006, P.R. China
}

Received June 13, 2013; Accepted July 18, 2013

DOI: $10.3892 / o r .2013 .2685$

\begin{abstract}
RNAs (miRNAs) are short noncoding RNAs, which modulate the expression of numerous genes by targeting mRNAs. Numerous abnormal miRNA expression patterns are found in various human malignancies, and certain miRNAs act as oncogenes or tumor suppressors. microRNA-155 (miR-155) may not only function as an oncogene but also as a tumor suppressor in various types of cancer cells, such as melanoma. Although miR-155 has been found to be upregulated in glioma, its role has not yet been eludicated in glioma tumorigenesis. Based on the prediction of the target genes of miR-155, we hypothesized that there is a significant association between miR-155 and FOXO3a, a negative regulator of Akt signaling. In the present study, we found that FOXO3a expression was significantly downregulated and miR-155 was upregulated in a panel of glioma cells and tissue specimens. Furthermore, we demonstrated that miR-155 induced cell proliferation by inhibiting apoptosis and promoted the migration and invasiveness of glioma cells, while miR-155 had no effect on the cell cycle as determined by gain-of-function and loss-of-function experiments. Moreover, we confirmed that miR-155 downregulated the expression of FOXO3a by directly targeting its 3'-UTR. These findings indicate that miR-155 may function as an oncogene by targeting FOXO3a in the development and progression of glioma.
\end{abstract}

Correspondence to: Dr Xiangdong Li, Department of Neurosurgery, The First Affiliated Hospital of Soochow University, 188 Shizi Street, Suzhou 215006, P.R. China

E-mail: xdlijia@yahoo.com.cn

Professor Hong-Tao Zhang, Soochow University Laboratory of Cancer Molecular Genetics, Medical College of Soochow University, 199 Ren'ai Road, Sino-Singapore Industrial Park, Suzhou 215123, P.R. China

E-mail: htzhang@suda.edu.cn

*Contributed equally

Key words: glioma, miR-155, FOXO3, proliferation, invasion

\section{Introduction}

Glioma is the most common primary malignant brain tumor in adults, and the prognosis and survival rate for patients with glioma are still extremely poor despite major therapeutic improvements (1). Particularly, glioblastoma multiforme is the most malignant form of glioma according to the current WHO classification of brain tumors $(2,3)$. Exploring the molecular mechanism involved in glioma tumorigenesis and progression has become an urgent demand for developing novel therapeutic strategies for glioma. Recent studies have reported the alteration of miRNAs in brain cancer cells, indicating their roles as oncogenes or tumor suppressors (4-6).

microRNAs (miRNAs) comprise a specialized subset of small cytoplasmic noncoding RNAs between 19 and 24 nucleotides in length. It was found that miRNAs play an important role in regulating various biological processes such as cell proliferation, differentiation, apoptosis, the cell cycle, migration, invasion, neuron development and tumorigenesis (4-6). miRNAs can function as tumor suppressors or oncogenes, depending on the target genes (7) and mainly exert their regulatory effects at the post-transcriptional level by targeting complementary or partly complementary mRNAs and inducing mRNA cleavage or translation repression (8). microRNA-155 (miR-155) is one of the miRNAs most frequently involved in cancers, and was initially implicated in the oncogenesis of hematopoietic malignancies based on the finding that BIC/ miR-155 expression is upregulated in B-cell lymphomas and chronic lymphocytic leukemia (9). miR-155 was also found to be overexpressed in various solid tumors, including lung, colon, breast, cervical, and thyroid cancers (10-13).

Of the mammalian Forkhead transcription factors, FOXO3a is one member that has emerged as a versatile target for a number of disorders, including various tumors. Previously, it has been reported that FOXO3a regulates oxidative detoxification, DNA damage response and stimulates DNA repair pathways $(14,15)$. With respect to apoptotic death, Foxo3a has been shown to modulate a ligand activating a Fas-mediated death pathway (16) and to induce tumor necrosis factor-related apoptosis-induced ligand (TRAIL) and the BH3-only proteins Noxa and Bim $(17,18)$. FOXO3a also can modulate growth 
arrest and DNA damage response protein 45 (Gadd45), influence cell cycle regulation $(19,20)$ and induce the repression of anti-apoptotic molecules FLIP and BCL-XL (21). Shiota et al demonstrated that FOXO3a may repress cancer cell aggressiveness through negative regulation of Twist/YB-1 signaling, in addition to its positive effects on E-cadherin in the progression of urothelial cancer (22).

Although miR-155 has been found to be upregulated in glioma, its role has not yet been defined in glioma tumorigenesis. Based on the prediction of target genes of miR-155, we hypothesized that there is a significant association between FOXO3a and miR-155. In the present study, we investigated the effect of miR-155 on the proliferation and invasion of glioma cells using loss-of-function and recovery experiments. Furthermore, we aimed to determine whether miR-155 could directly bind to FOXO3a. The results suggest that miR-155 serves as an oncomiR and a regulator of FOXO3a expression in glioma.

\section{Materials and methods}

Cell culture and human tissue samples. The glioma cell line U251 was obtained from the Cell Bank of the Chinese Academy of Sciences (Shanghai, China). U87, U373, SHG44 and T98 cells were gifts from the Neuroscience Institute of Soochow University. The normal human astrocyte 1800 cell line was obtained from the American Type Culture Collection (Manassas, VA, USA). All cells were cultured at $37^{\circ} \mathrm{C}$ in a humidified atmosphere containing $5 \% \mathrm{CO}_{2}$ and maintained in Dulbecco's modified Eagle's medium (DMEM; Gibco, Paisley, UK) supplemented with $10 \%$ heat inactivated fetal bovine serum (FBS; Gibco, USA), $100 \mu \mathrm{g} / \mathrm{ml}$ streptomycin and $1 \mathrm{U} /$ $\mathrm{ml}$ penicillin.

Fresh glioma specimens were obtained with written informed consent from 7 patients with glioma who underwent surgery at the First Affiliated Hospital of Soochow University. None of the patients had received radiotherapy or chemotherapy prior to surgery. Two normal brain tissue samples were obtained from patients who underwent surgery for cerebral trauma. The resected tissue samples were immediately snap frozen in liquid nitrogen until use. All human materials were used in accordance with the policies of the local institutional review boards.

Real-time PCR. Total RNA was extracted with TRIzol (Invitrogen, Oslo, Norway). For mature microRNA expression analysis, miR-155 was detected using an All-in-One ${ }^{\mathrm{TM}}$ miRNA qRT-PCR Detection kit (GeneCopoeia, Rockville, MD, USA) according to the manufacturer's instructions. For mRNA, total RNA isolated from glioma samples and cells were subsequently reverse transcribed to cDNA using All-in-One First-Strand cDNA Synthesis kit (GeneCopoeia). The synthesized cDNA was then used to amplify gene regions by quantitative PCR using the All-in-One qPCR mix (GeneCopoeia catalog no. AOPR-0200). The U6 small nuclear RNA and GAPDH mRNA were used as internal controls for miR-155 and FOXO3a mRNA, respectively. Relative expression was calculated using the $\Delta \Delta \mathrm{Ct}$ method. The reactions were placed in a 96-well plate (ABI) using the 7500 Real-Time PCR system (Applied Biosystems).
Western blot analysis. Protein of the treated cell lines was extracted by mammalian protein extraction reagent (Pierce, USA) supplemented with protease inhibitors and phosphatase inhibitor cocktail (Sigma, USA). Protein $(25 \mu \mathrm{g})$ samples were separated on a $10 \%$ SDS-PAGE gel and then transferred to PVDF membranes. After blocking with 1\% BSA (Amresco, USA), the membranes were incubated with antibodies against FOXO3a (75D; Cell Signaling, Danvers, MA, USA) diluted 1:1000 or $\beta$-actin (Santa Cruz Biotechnology, Inc., Santa Cruz, CA, USA) diluted 1:2000 overnight at $4^{\circ} \mathrm{C}$; the membrane was washed and then incubated with horseradish peroxidase (HRP)conjugated goat anti-rabbit (Dako, Glostrup, Denmark) or goat anti-mouse (Santa Cruz Biotechnology, Inc.) secondary antibody diluted 1:2000 for $2 \mathrm{~h}$. The membrane was washed again, and the antigen-antibody reaction was visualized using an ECL detection system. All experiments were repeated in triplicate.

miRNA transfection. miR-155 mimics, miR-155 inhibitor and the respective negative control (miR-NC and anti-NC) were designed and synthesized by GenePharma (Shanghai, China). The miR-155 inhibitor is single-stranded RNA molecules, which can specifically knock down endogenous miR-155. Cells were transfected using Entranster-R (Engreen Biosystem Co., Ltd., China). Transfection complexes were prepared according to the manufacturer's instructions and added directly to the cells in serum medium. miR-155 mimics and miR-NC were transfected to U251 cells, which expressed low levels of miR-155; miR-155 inhibitor (anti-miR-155), inhibitor $\mathrm{NC}$ (anti-NC) were transfected to U87 cells, which expressed high levels of miR-155.

Cell proliferation. Cell counts were determined using Cell Counting Kit-8 (Dojindo, Japan). Three thousand cells per well were seeded in a 96-well plate and incubated for $24 \mathrm{~h}$, and the cells were transfected with miR-155 mimics, miR-155 inhibitor or $\mathrm{NC}$ at the final concentration of $50 \mathrm{nM} / \mu 1$. Ten microliters of Cell Counting Kit-8 was added to $100 \mu \mathrm{l}$ cell culture media and incubated for $2 \mathrm{~h}$ in $\mathrm{CO}_{2}$ incubator at 12 , 24,48 and $72 \mathrm{~h}$ after transfection. Absorbance was measured at $450 \mathrm{~nm}$. Three independent experiments were performed.

Cell cycle analysis. Cell cycle distribution was assessed using a flow cytometer (FC500 Flow Cytometer; Beckman Coulter, USA). According to the manual from the cell cycle analysis kit (Beyotime, China), cells were allowed to inoculate and adhere for $24 \mathrm{~h}$ in 6 -well plates. Then miR-155 mimics, miR-155 inhibitor and $\mathrm{NC}$ were transfected into cells of the respective well, and cells were incubated for a further period of $24 \mathrm{~h}$, then trypsinized, washed three times in cold phosphate-buffered saline (PBS), fixed in $70 \%$ absolute ice-cold ethanol, kept at $4{ }^{\circ} \mathrm{C}$ overnight and stained in a mixture of propidium (PI) RNase A; the stained cells were analyzed using flow cytometry. This experiment was repeated three times.

Cell apoptosis assay. Cells were transfected with miR-155, antimiR-155, or NC at $50 \%$ confluence. After $48 \mathrm{~h}$, the adherent cells were harvested by trypsinization and were washed with PBS once and resuspended in $500 \mu \mathrm{l}$ of $1 \mathrm{X}$ binding buffer (Annexin V/FITC kit; Sigma). Then, $10 \mu 1$ Annexin V/FITC and $5 \mu \mathrm{l}$ propidium iodide were added into the binding buffer, 
A

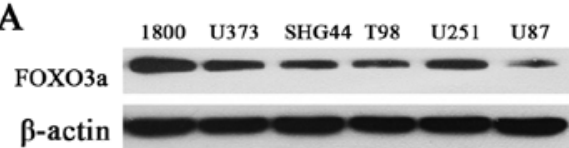

C

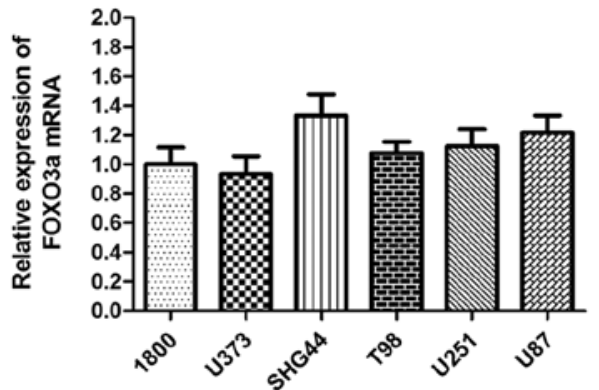

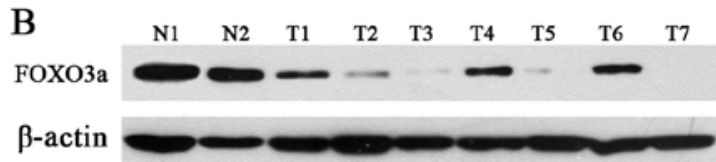

D

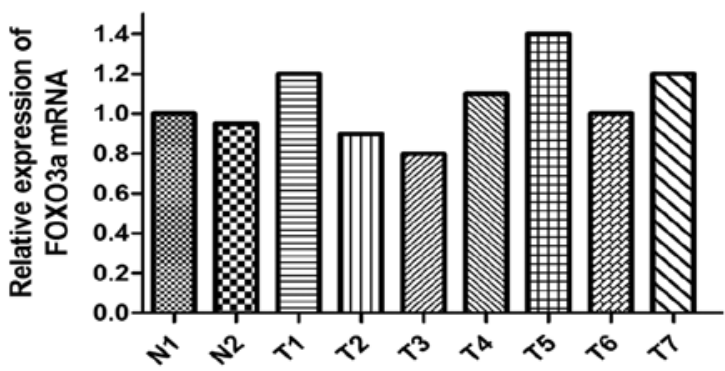

Figure 1. Expression of FOXO3a in glioma cell lines and human glioma tissues. Western blot analysis showed that levels of FOXO3a in human glioma cell lines (U373, SHG44, T98, U251 and U87) (A) and glioma tissue specimens (B) were significantly lower than those in the astrocyte 1800 cell line and human normal brain tissue samples, respectively. As a normalizing control, $\beta$-actin was also detected in the same blot. Real-time PCR assays showed no statistical significant difference in FOXO3a mRNA expression between glioma cell lines and the normal astrocyte 1800 cell line (C) or between glioma tissue specimens and/or normal brain tissue (D).

and the tubes were incubated at room temperature for exactly $10 \mathrm{~min}$ in the dark. The fluorescence of the cells was immediately determined with a flow cytometer.

Monolayer wound healing assay. Migratory ability was determined using a wound healing assay. Cells were grown in $10 \%$ FBS medium on 6-well plates. After the cells reached sub-confluence, the cells were wounded by scraping the monolayer with a $200-\mu 1$ pipette tip and grown in $1 \%$ FBS medium. The floating cells were removed by gentle washes with culture medium. The healing process was dynamically examined and was recorded with a digital camera at $24 \mathrm{~h}$ after the wound was created.

Transwell invasion assay. For the Transwell assay, $2 \times 10^{4}$ transfected cells were plated into 24-well Boyden chambers (Corning Costar, Cambridge, MA, USA), with an $8-\mu \mathrm{m}$ pore polycarbonate membrane, which was coated with $30 \mu \mathrm{g}$ of Matrigel (BD Biosciences, San Jose, CA, USA). Cells were in the upper chamber with $200 \mu \mathrm{l}$ of serum-free medium, and medium containing $20 \%$ FBS was added to the lower chamber to serve as a chemoattractant. After $36 \mathrm{~h}$, the cells were washed 3 times with PBS. Non-invasive cells were removed from the upper well by cotton swabs, and the invasive cells were then fixed with paraformaldehyde for $15 \mathrm{~min}$, air-dried, and stained with $0.1 \%$ crystal violet for $15 \mathrm{~min}$. The cells were recorded with a digital camera.

Luciferase activity assay. DNA fragments from 3'-UTR of FOXO3a that host the predicted complementary sites of miR-155 were cloned to downstream of the Renilla luciferase reporter gene in psiCHECK2 dual luciferase reporter plasmid (Promega, Madison, WI, USA). We amplified a 2192-bp FOXO3a 3'-UTR region containing four tandem repeats of miR-155 response element, which includes the artificial XhoI and NotI enzyme restriction sites with forward primer 5'-TGTTGTTCTTGTG
TTTGTTTTCC-3' and reverse primer 5'-ATTCTCCTGATC TGTTTTGTGCT-3'. The amplified fragments were then cleaved with the XhoI and NotI enzymes (New England BioLabs, Ipswich, MA, USA) as well as psiCHECK2 vector (Promega), and the above-prepared fragment and psiCHECK2 vector were then ligated by T4 DNA ligase (New England BioLabs). The recombined vector was named psi-FOXO3a.

$\mathrm{U} 251$ or U87 cells were seeded at $1 \times 10^{5}$ cells per well in 24-well plates. At $24 \mathrm{~h}$ after the plating, cells were transfected by Lipofectamine 2000 (Invitrogen, Carlsbad, CA, USA) according to the manufacturer's instructions. In each well, $800 \mathrm{ng}$ psi-FOXO3a vectors were co-transfected with $50 \mathrm{pmol}$ miR-155 mimics, miR-155 inhibitors or NC accordingly. There were four replicates for each group, and the experiment was repeated at least three times. At $24 \mathrm{~h}$ after transfection, cells were harvested by passive adding of $100 \mu \mathrm{l}$ buffer. Renilla luciferase activities in the cell lysate were measured with the Dual-Luciferase Reporter assay system (Promega) in TD-20/20 luminometer (Turner Biosystems, Sunnyvale, CA, USA) and were normalized to the firefly luciferase activities.

Statistical analysis. Data shown in the graphs represent the mean values \pm SD of three independent experiments performed. The difference among groups was determined by ANOVA analysis and comparison between two groups was analyzed by the Student's t-test using GraphPad Prism software version 4.0 (GraphPad Software, Inc., San Diego, CA, USA). A value of $\mathrm{P}<0.05$ was considered statistically significant.

\section{Results}

Expression of $\mathrm{FOXO3a}$ in glioma cell lines and human glioma tissues. To explore the role of FOXO3a in glioma, we used western blotting to detect the expression level of FOXO3a in glioma cell lines, human glioma and normal brain tissues. The findings showed that five carcinoma cell lines (U251, U87, 

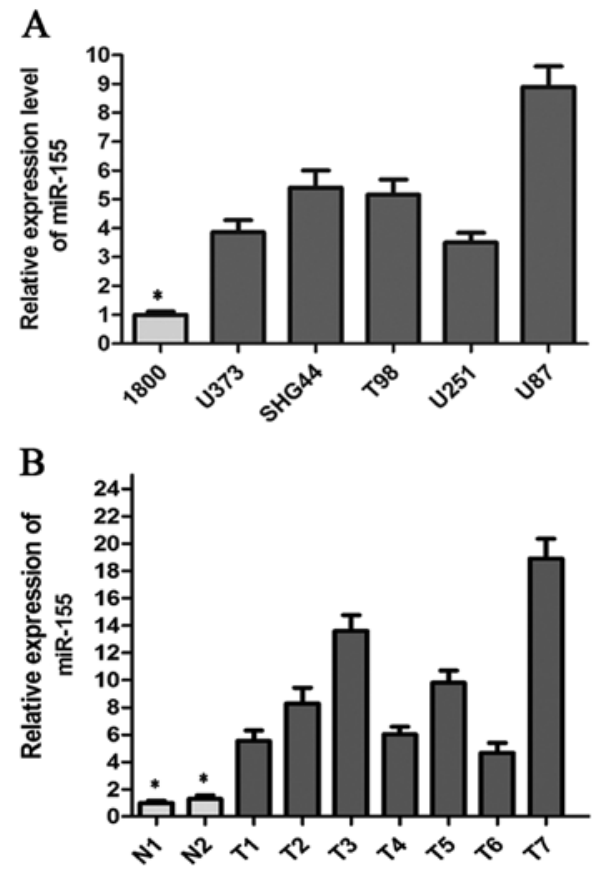

Figure 2. Detection of miR-155 expression in glioma cell lines and tumor tissues using real-time PCR. (A) The miR-155 expression level was significantly higher in glioma cell lines when compared with that in the normal human astrocyte 1800 cell line. (B) miR-155 expression was upregulated in the human glioma tissues. The data were derived from three independent measurements. ${ }^{*} \mathrm{P}<0.05$.

U373, T98 and SHG44) presented lower expression of FOXO3a protein when compared with the normal astrocyte 1800 cell line (Fig. 1A). Among all glioma cell lines, the highest expression of FOXO3a was detected in U251 cells, while the lowest expression was observed in U87 cells. Moreover, FOXO3a levels in glioma tissue were significantly lower than those in normal brain tissues (Fig. 1B). However, no significant difference in FOXO3a mRNA was observed between glioma cell lines, and between human glioma tissues, respectively (Fig. 1C and D).

miR-155 is upregulated in glioma cell lines and the glioma samples. To determine whether miR-155 plays a role in glioma tumorigenesis, we conducted real-time quantitative PCR to quantify mature miR-155 in five glioma cell lines (U251, U87, U373, T98 and SHG44) and seven glioma tissue samples, using the normal human astrocyte 1800 cell line and two normal brain tissue samples as corresponding controls. As shown in Fig. 2A, miR-155 expression was notably upregulated in five glioma cell lines, when compared to that in the normal astrocyte 1800 cell line. Among all glioma cell lines, the U87 cell line expressed the highest level of miR-155 (9-fold compared to the normal control) and U251 cell line expressed the lowest level of miR-155 (3.5-fold compared to the normal control) (Fig. 2A). Higher expression of miR-155 was observed in all glioma tissue samples (Fig. 2B).

FOXO3a is a direct target of miR-155. TargetScan prediction in silico and previous studies indicated that FOXO3a is the theoretical target gene of miR-155 (Fig. 3A). To test this possibility, fragments of 3'-UTR of human FOXO3a containing
A
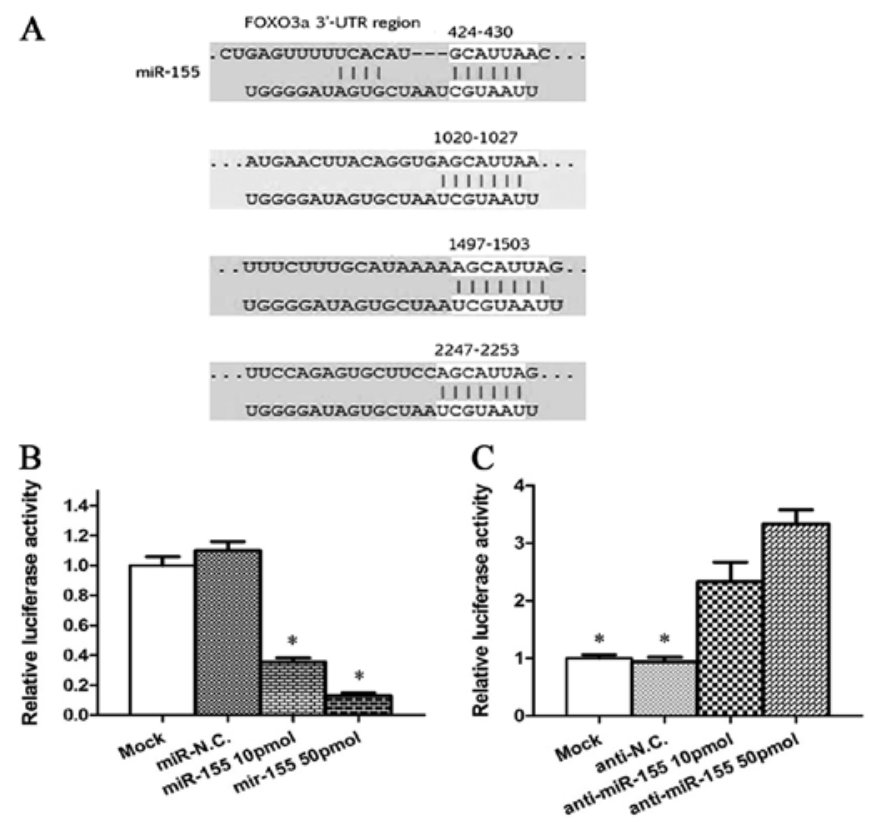

D
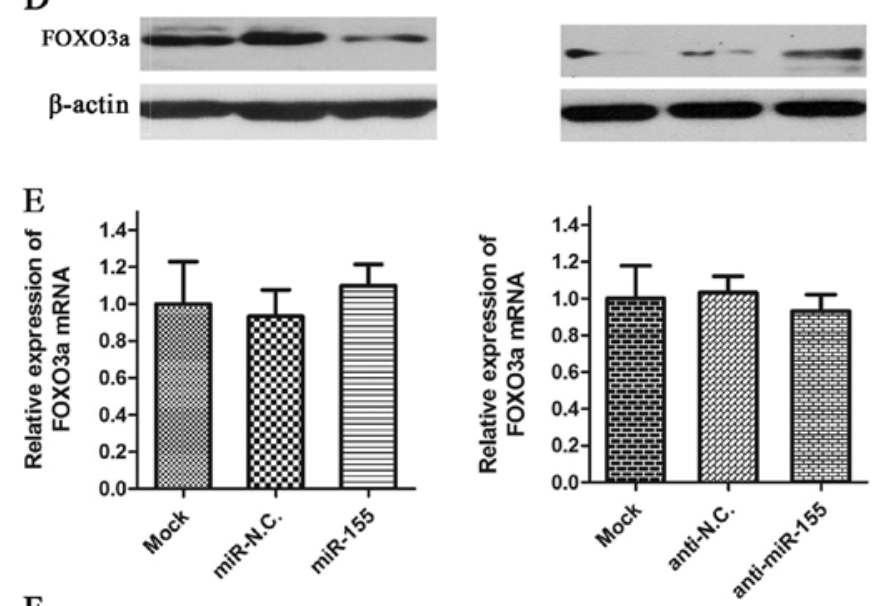

$\mathrm{F}$
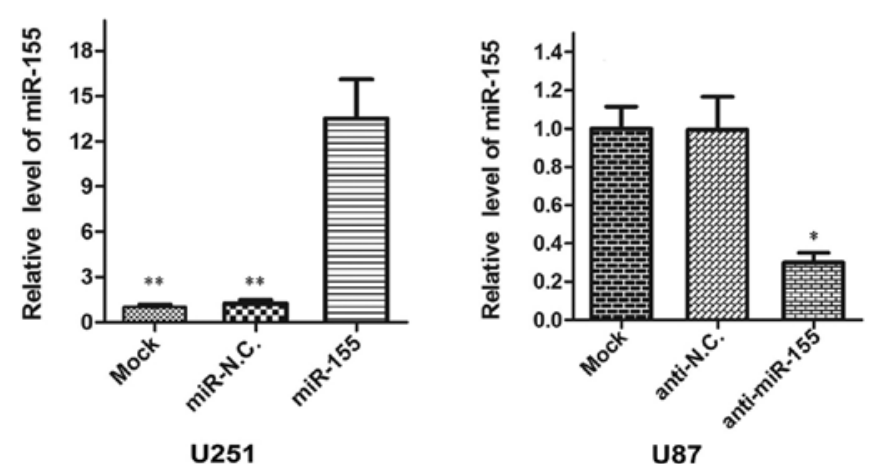

Figure 3. miR-155 directly inhibits FOXO3a expression. (A) Schematic graph of the putative four binding sites of miR-155 in the FOXO3a 3'-UTR. (B and C) Dual luciferase reporter assay was performed by transiently transfecting U251 and U87 cells with psi-FOXO3a-3'-UTR and then by co-transfecting them with miR-155 mimics or miR-155 inhibitors, respectively. When the psi-FOXO3a vector was co-transfected with miR-155, the luciferase activity of vector was significantly decreased compared with that co-transfected with miR-NC and Mock. As expected, the luciferase levels of psi-FOXO3a-3'-UTR in U87 cells were restored after transfection with miR-155 inhibitors. (D and E) Detection of FOXO3a protein and mRNA expression in U251 cells following trasnfection with miR-155 mimics and in U87 cells following transfection with miR-155 inhibitors, respectively. Overexpression of miR-155 reduced expression of the FOXO3a protein but not FOXO3a mRNA. (F) miR-155 mimics (miR-155) or miR-155 inhibitors (anti-miR-155) significantly altered the expression of miR-155 at $48 \mathrm{~h}$ after transfection when compared with miR-NC and Mock. "P<0.05, ${ }^{\text {"* }} \mathrm{P}<0.01$. 
A

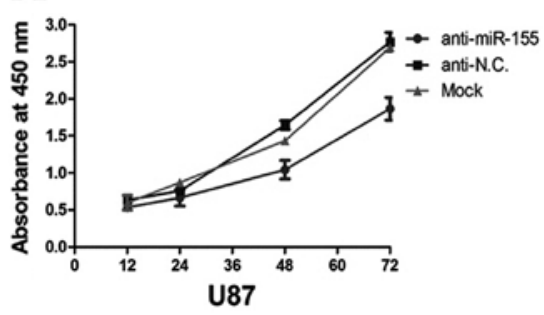

C

U87

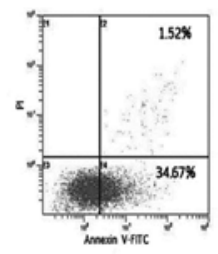

Anti-miR-155

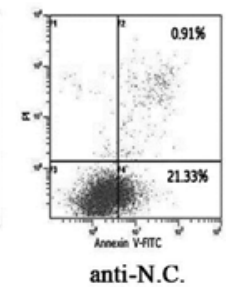

D

$\mathrm{U} 251$

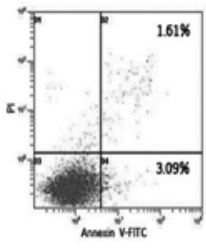

miR-155

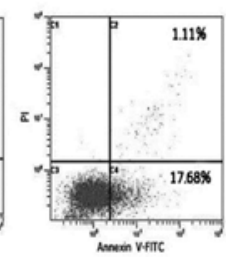

miR-N.C.
B
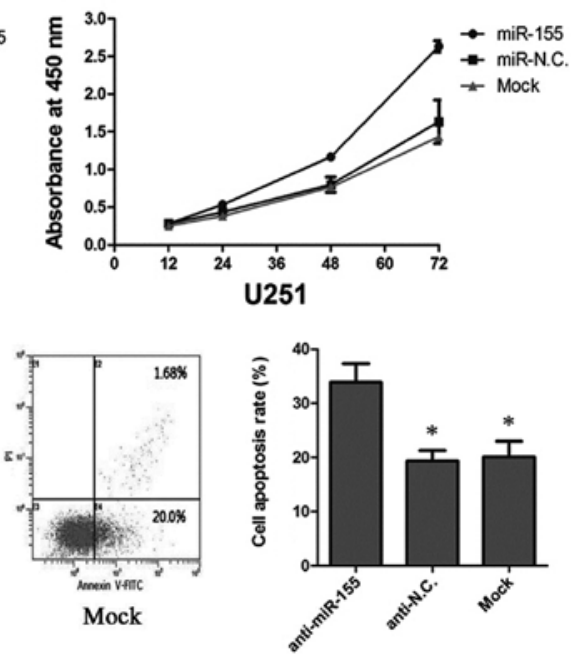

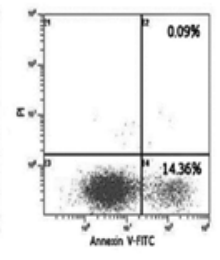

Mock

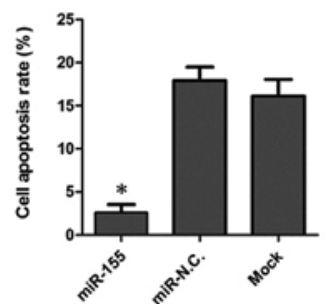

E

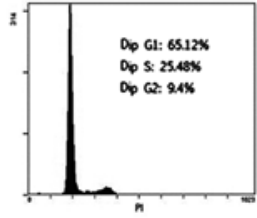

Anti-miR-155

F

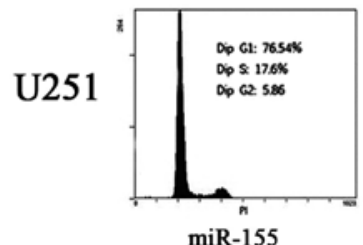

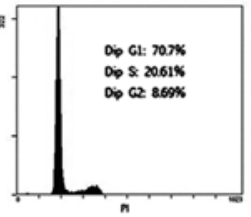

anti-N.C.

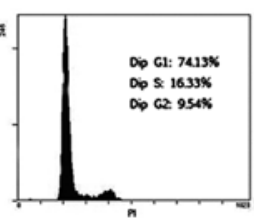

miR-N.C.

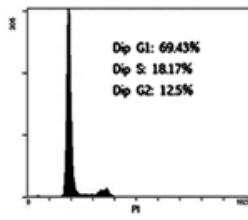

Mock

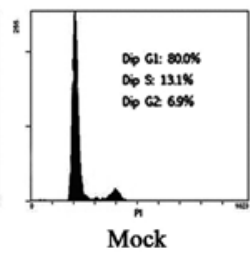

Figure 4. Effect of miR-155 on cell proliferation, cell cycle distribution and apoptosis. (A and B) Cell proliferation was dramatically decreased in U87 cells after transfection with the miR-155 inhibitor, while the proliferation was increased in U251 cells after transfection with miR-155 mimics. (C and D) Effect of the alteration of miR-155 expression on cell apoptosis as detected by flow cytometric analysis in U87 and U251 cells. Inhibition of miR-155 increased the rate of apoptosis. ${ }^{\mathrm{P}}<0.05$. (E and F) Flow cytometric analysis of the effect of miR-155 on the cell cycle of U87 and U251 cells. The alteration of miR-155 expression was ineffective on the cell cycle distribution of glioma cells.

miR-155 complementary sites were cloned into psiCHECK2 dual luciferase reporter plasmid. When the psi-FOXO3a vector was co-transfected with miR-155, the luciferase activity of the vector was dramatically decreased $(\mathrm{P}<0.05)$ compared with those co-transfected with miR-NC and Mock in U251 cells (Fig. 3B). As expected, the luciferase levels of psi-FOXO3a3'-UTR in U87 cell lines were restored after transfection with the miR-155 inhibitor (Fig. 3C).

By performing western blot analysis, a pronounced reduction in FOXO3a protein level was noted in the U251 cells in which miR-155 is overexpressed and an increase in U87 cells in which miR-155 is downregulated (Fig. 3D). However, no significant difference in FOXO3a mRNA was observed between cells overexpressing/underexpressing miR-155 and those with NC (Fig. 3E and F). This raised the possibility that miR-155 suppresses FOXO3a protein synthesis by a post-transcriptional repression mechanism, via its 3'-UTR complementary sites.

miR-155 increases cell proliferation by inhibiting apoptosis in glioma cells. In order to explore the functional role of miR-155 on the growth of glioma cells, we performed cellular proliferation assays (CCK-8 assay) in U87 and U251 cells and found that cell proliferation was dramatically decreased in U87 cells after transfection with the miR-155 inhibitor, while proliferation was increased in U251 cells transfected with miR-155 mimics $(\mathrm{P}<0.05)$ (Fig. 4A and B). Then, we carried out cell apoptosis and cell cycle assays to examine whether the reduction in cell 


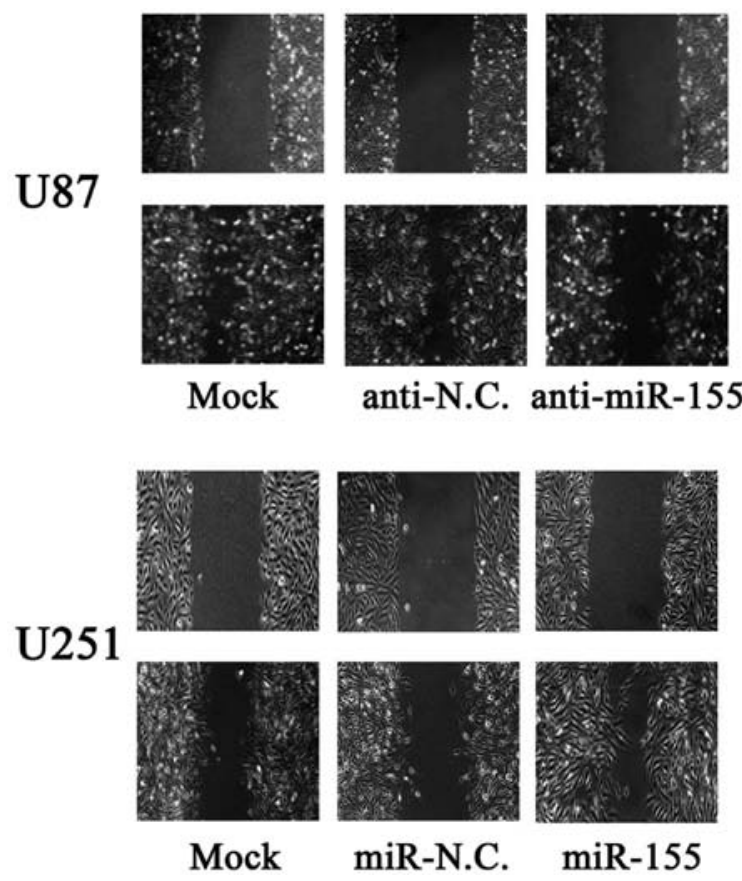

Figure 5. Effect of miR-155 expression on the migration of glioma cells. Wound healing assay was performed on U87 cells transfected with miR-155 mimics and miR-NC, and on U251 cells transfected with miR-155 inhibitors and inhibitor-NC. After $24 \mathrm{~h}$, U87 cells transfected with miR-155 inhibitors closed the wound more slowly than NC, while U251 cells transfected with miR-155 mimics obtained the opposite results.

proliferation was due to increased apoptosis or attenuated cell cycle induced by downregulation of miR-155. The U87 cells transfected with the miR-155 inhibitor were found to exhibit an enhanced apoptosis rate, compared to the anti-NC and Mock groups, while U251 cells transfected with miR-155 mimics exhibited the opposite results (Fig. 4C and D), while the alteration of miR-155 expression was ineffective on the cell cycle distribution of glioma cells (Fig. 4E and F). These results suggest that miR-155 increases the cell proliferative ability by inhibiting apoptosis in glioma cells.

miR-155 promotes the migration and invasion of glioma cells. To investigate whether miR-155 has a direct functional role in facilitating glioma cell migration and invasion, we evaluated cancer cell invasion through Transwell assay and assessed migration through a wound healing assay. As shown in Fig. 5, inhibition of miR-155 impeded the migration of U87 cells by $\sim 50 \%$ when compared with the control. Similarly, invasion of U87 cells was reduced by $59 \%$ following inhibition of miR-155 (Fig. 6). Conversely, transfection of U251 cells with miR-155 mimics promoted cell migration and invasion ability by 2 -fold (Fig. 5 and 6). These data suggest that miR-155 is an onco-miRNA that can promote cell migration and invasion in glioma cells.

\section{Discussion}

miR-155 has emerged as an essential regulator of cellular physiology, and deregulation of miR-155 is implicated in a wide range of diseases, including pituitary adenoma, multiple malignant tumors, myelodysplastic syndrome and Down
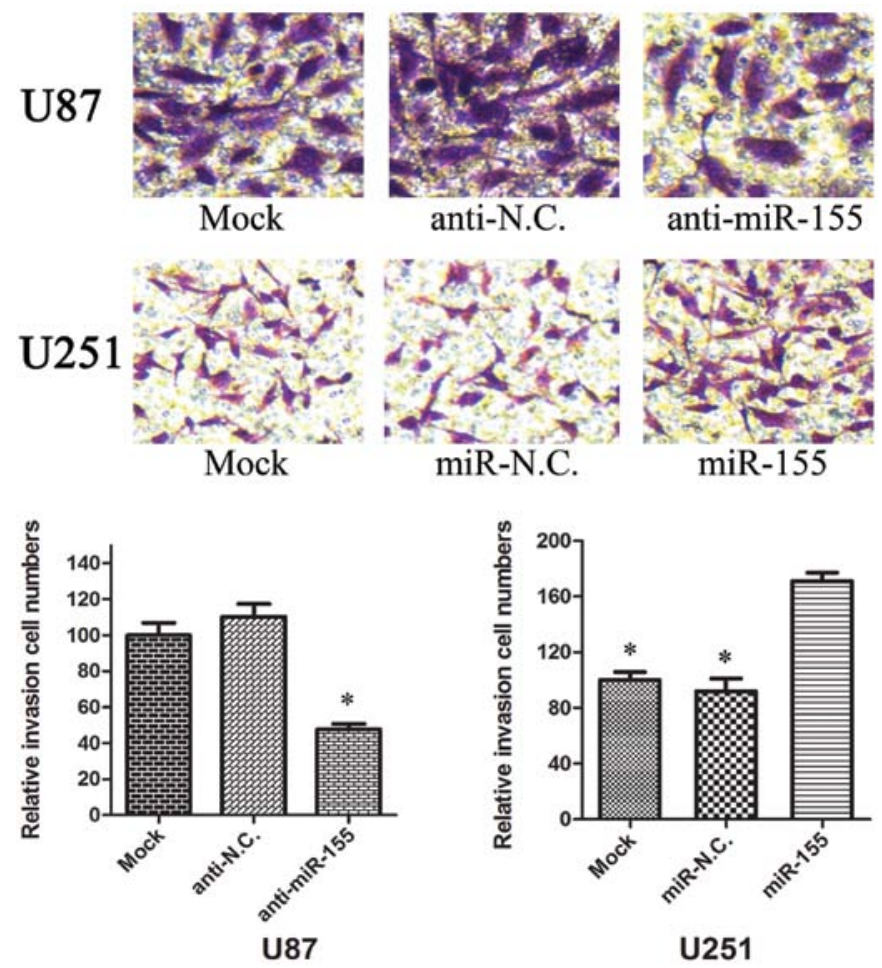

Figure 6. Effect of miR-155 expression on the invasion of glioma cells Transwell assay was performed on the U87 cells transfected with miR-155 mimics and miR-NC, and on U251 cells transfected with miR-155 inhibitors and inhibitor-NC. U251 cells transfected with miR-155 and NC invading to the underside of the Matrigel-coated insert membrane were fixed and stained. Cell numbers were counted and the respective histograms indicate that a higher number of cells transfected with miR-155 invaded to the membrane than the number of cells in the NC and Mock groups. U87 cells transfected with miR-155 inhibitors exhibited the opposite results. ${ }^{*} \mathrm{P}<0.05$.

syndrome (23-26). miR-155 has been extensively investigated in immunology and lymphoma; however, it is only evident that miR-155 expression is increased in glioma, and to date the detailed function of miR-155 remains elusive. In the present study, we also found that miR-155 was upregulated in glioma cell lines and all glioma tissue samples, and we transfected the miR-155 inhibitor and miR-155 mimics into U87 and U251 cells, repectively, to evaluate glioma cell proliferation, the cell cycle, apoptosis, migration and invasion. The results suggest that miR-155 may not only enhance cell proliferative ability by inhibiting apoptosis but also may promote cell migration and invasion in glioma cells.

To further elucidate the molecular mechanisms and improve our understanding of the regulatory function of miR-155 in glioma, we first analyzed the expression of FOXO3a, which was predicated as a target of miR-155, and found that expression of FOXO3a protein was markedly attenuated in glioma cells and human glioma tissues. It was reported that inactivation of FOXO3a in glioma cells led to the downregulation of Bim, a pro-apoptotic protein (20). Hu and colleagues demonstrated that expression of FOXO3a exerts an inhibitory effect on tumorigenesis and cell growth in breast cancer cells (27). In cancer cells, suppression of FOXO3a was believed to be due mainly to the activation of multiple onco-kinases such as Akt, SGK and IKK. However, the dysregulation of FOXO3a can be caused by various mechanisms, including miRNA regulation. 
Subsequently, we used two different methods to determine whether FOXO3a is a bonafide target of miR-155. Overexpression of miR-155 caused the reduction in FOXO3a protein albeit there was no difference in FOXO3a mRNA expression between miR-155-overexpressing and negative control cells. These results are consistent with previous data, which indicated that miR-155-induced silencing appears to occur only through translational repression without affecting mRNA levels (28). Furthermore, the luciferase activity assay demonstrated that downregulation of FOXO3a was mediated by miR-155, which may target 3'-UTR of FOXO3a. Thus, our findings support the hypothesis that the effect of miR-155 on proliferation and invasion of glioma cells may be attributed to the downregulation of FOXO3a, via directly targeting the FOXO3a-3'-UTR.

However, an opposite trend in the expression of miR-155 has been observed in other types of cancer. For example, miR-155 was found to be downregulated in melanoma when compared with non-cancerous tissue and could mediate melanoma growth inhibition via SKI gene silencing (29). As exemplified by miR-10b, it was found to be overexpressed in glioma and induced glioma cell invasion by modulating tumor invasion factors MMP-14 and uPAR expression via the direct target HOXD10 (30). However, Moriarty et al found that miR-10b was downregulated and inhibited Tiam1-mediated Rac activation to suppress migration and invasion of breast carcinoma (31). In addition, overexpression of miR-30 inhibited apoptosis via suppression of p53 expression in cardiomyocytes (32), while upregulation of miR-30 induced apoptosis by targeting Ubc9 in breast cancer cells (33), suggesting that a single miRNA may have several distinct functions in different cell types, which likely depends on the availability of specific targets or downstream effectors. Therefore, it is extremely important to validate targets of miR-155 by further functional assays.

In summary, we demonstrated that miR-155 is markedly upregulated in human glioma, and is able to downregulate FOXO3a expression to enhance the proliferative and invasive ability of glioma cells, suggesting that silencing of miR-155 expression may be a novel strategy for the treatment of human glioma.

\section{Acknowledgements}

This study was supported in part by grants from the Program for New Century Excellent Talents in University (NCET-090165 to H.-T. Zhang), Jiangsu Province's Key Provincial Talents Program (RC2011106 to J. Zhao), '333' Project of Jiangsu Province Government (to H.-T. Zhang), Soochow Scholar Project of Soochow University (to H.-T. Zhang), and Suzhou Key Laboratory for Molecular Cancer Genetics (SZS201209 to H.-T. Zhang).

\section{References}

1. Behin A, Hoang-Xuan K, Carpentier AF and Delattre JY: Primary brain tumours in adults. Lancet 361: 323-331, 2003.

2. Zhou H, Miki R, Eeva M, Fike FM, Seligson D, Yang L, Yoshimura A, Teitell MA, Jamieson CA and Cacalano NA: Reciprocal regulation of SOCS 1 and SOCS3 enhances resistance to ionizing radiation in glioblastoma multiforme. Clin Cancer Res 13: 2344-2353, 2007.
3. Ohgaki H: Genetic pathways to glioblastomas. Neuropathology 25: 1-7, 2005

4. Iorio MV and Croce CM: MicroRNAs in cancer: small molecules with a huge impact. J Clin Oncol 27: 5848-5856, 2009.

5. Brennecke J, Hipfner DR, Stark A, Russell RB and Cohen SM: bantam encodes a developmentally regulated microRNA that controls cell proliferation and regulates the proapoptotic gene hid in Drosophila. Cell 113: 25-36, 2003.

6. D'Urso PI, D'Urso OF, Storelli C, Mallardo M, Gianfreda CD, Montinaro A, Cimmino A, Pietro C and Marsigliante S: miR-155 is up-regulated in primary and secondary glioblastoma and promotes tumour growth by inhibiting GABA receptors. Int J Oncol 41: 228-234, 2012.

7. Lee KH, Chen YL, Yeh SD, Hsiao M, Lin JT, Goan YG and Lu PJ MicroRNA-330 acts as tumor suppressor and induces apoptosis of prostate cancer cells through E2F1-mediated suppression of Akt phosphorylation. Oncogene 28: 3360-3370, 2009.

8. Yekta S, Shih IH and Bartel DP: MicroRNA-directed cleavage of HOXB8 mRNA. Science 304: 594-596, 2004.

9. Faraoni I, Antonetti FR, Cardone J and Bonmassar E: miR-155 gene: a typical multifunctional microRNA. Biochim Biophys Acta 1792: 497-505, 2009.

10. Yanaihara N, Caplen N, Bowman E, Seike M, Kumamoto K, Yi M, Stephens RM, Okamoto A, Yokota J, Tanaka T, Calin GA, Liu CG, Croce CM and Harris CC: Unique microRNA molecular profiles in lung cancer diagnosis and prognosis. Cancer Cell 9: 189-198, 2006.

11. Yip L, Kelly L, Shuai Y, Armstrong MJ, Nikiforov YE, Carty SE and Nikiforova MN: MicroRNA signature distinguishes the degree of aggressiveness of papillary thyroid carcinoma. Ann Surg Oncol 18: 2035-2041, 2011.

12. Wang X, Tang S, Le SY, Lu R, Rader JS, Meyers C and Zheng ZM: Aberrant expression of oncogenic and tumor-suppressive microRNAs in cervical cancer is required for cancer cell growth. PLoS One 3: e2557, 2008.

13. Iorio MV, Ferracin M, Liu CG, Veronese A, Spizzo R, Sabbioni S, Magri E, Pedriali M, Fabbri M, Campiglio M, Menard S, Palazzo JP, Rosenberg A, Musiani P, Volinia S, Nenci I, Calin GA, Querzoli P, Negrini M and Croce CM: MicroRNA gene expression deregulation in human breast cancer. Cancer Res 65: 7065-7070, 2005.

14. Tran H, Brunet A, Grenier JM, Datta SR, Fornace AJ Jr, DiStefano PS, Chiang LW and Greenberg ME: DNA repair pathway stimulated by the forkhead transcription factor FOXO3a through the Gadd45 protein. Science 296: 530-534, 2002.

15. Tsai WB, Chung YM, Takahashi Y, Xu Z and Hu MC: Functional interaction between FOXO3a and ATM regulates DNA damage response. Nat Cell Biol 10: 460-467, 2008.

16. Barthelemy C, Henderson CE and Pettmann B: Foxo3a induces motoneuron death through the Fas pathway in cooperation with JNK. BMC Neurosci 5: 48, 2004.

17. Obexer P, Geiger K, Ambros PF, Meister B and Ausserlechner MJ: FKHRL1-mediated expression of Noxa and Bim induces apoptosis via the mitochondria in neuroblastoma cells. Cell Death Differ 14: 534-547, 2007.

18. Delpuech O, Griffiths B, East P, Essafi A, Lam EW, Burgering B, Downward J and Schulze A: Induction of Mxil-SR alpha by FOXO3a contributes to repression of Myc-dependent gene expression. Mol Cell Biol 27: 4917-4930, 2007.

19. Furukawa-Hibi Y, Yoshida-Araki K, Ohta T, Ikeda K and Motoyama N: FOXO forkhead transcription factors induce G(2)-M checkpoint in response to oxidative stress. J Biol Chem 277: 26729-26732, 2002.

20. Brunet A, Bonni A, Zigmond MJ, Lin MZ, Juo P, Hu LS, Anderson MJ, Arden KC, Blenis J and Greenberg ME: Akt promotes cell survival by phosphorylating and inhibiting a Forkhead transcription factor. Cell 96: 857-868, 1999.

21. Tang TT, Dowbenko D, Jackson A, Toney L, Lewin DA, Dent AL and Lasky LA: The forkhead transcription factor AFX activates apoptosis by induction of the BCL- 6 transcriptional repressor. J Biol Chem 277: 14255-14265, 2002.

22. Shiota M, Song Y, Yokomizo A, Kiyoshima K, Tada Y, Uchino H, Uchiumi T, Inokuchi J, Oda Y, Kuroiwa K, Tatsugami K and Naito S: Foxo3a suppression of urothelial cancer invasiveness through Twist1, Y-box-binding protein 1, and E-cadherin regulation. Clin Cancer Res 16: 5654-5663, 2010.

23. Butz H, Liko I, Czirjak S, Igaz P, Khan MM, Zivkovic V, Balint K, Korbonits M, Racz K and Patocs A: Down-regulation of Weel kinase by a specific subset of microRNA in human sporadic pituitary adenomas. J Clin Endocrinol Metab 95: E181-E191, 2010. 
24. Xie Q, Chen X, Lu F, Zhang T, Hao M, Wang Y, Zhao J, McCrae MA and Zhuang $\mathrm{H}$ : Aberrant expression of microRNA 155 may accelerate cell proliferation by targeting sex-determining region Y box 6 in hepatocellular carcinoma. Cancer 118: 2431-2442, 2012.

25. Kuhn DE, Nuovo GJ, Terry AV Jr, Martin MM, Malana GE, Sansom SE, Pleister AP, Beck WD, Head E, Feldman DS and Elton TS: Chromosome 21-derived microRNAs provide an etiological basis for aberrant protein expression in human Down syndrome brains. J Biol Chem 285: 1529-1543, 2010.

26. Santamaria C, Muntion S, Roson B, Blanco B, Lopez-Villar O, Carrancio S, Sanchez-Guijo FM, Diez-Campelo M, AlvarezFernandez S, Sarasquete ME, de las Rivas J, Gonzalez M, San Miguel JF and Del Canizo MC: Impaired expression of DICER, DROSHA, SBDS and some microRNAs in mesenchymal stromal cells from myelodysplastic syndrome patients. Haematologica 97: 1218-1224, 2012.

27. Hu MC, Lee DF, Xia W, Golfman LS, Ou-Yang F, Yang JY, Zou Y, Bao S, Hanada N, Saso H, Kobayashi R and Hung MC: IkappaB kinase promotes tumorigenesis through inhibition of forkhead FOXO3a. Cell 117: 225-237, 2004.

28. Kong W, He L, Coppola M, Guo J, Esposito NN, Coppola D and Cheng JQ: MicroRNA-155 regulates cell survival, growth, and chemosensitivity by targeting FOXO3a in breast cancer. J Biol Chem 285: 17869-17879, 2010.
29. Levati L, Pagani E, Romani S, Castiglia D, Piccinni E, Covaciu C, Caporaso P, Bondanza S, Antonetti FR, Bonmassar E, Martelli F, Alvino E and D'Atri S: MicroRNA-155 targets the SKI gene in human melanoma cell lines. Pigment Cell Melanoma Res 24: 538-550, 2011.

30. Sun L, Yan W, Wang Y, Sun G, Luo H, Zhang J, Wang X, You Y, Yang $Z$ and Liu N: MicroRNA-10b induces glioma cell invasion by modulating MMP-14 and UPAR expression via HOXD10. Brain Res 1389: 9-18, 2011.

31. Moriarty CH, Pursell B and Mercurio AM: miR-10b targets Tiam1: implications for Rac activation and carcinoma migration. J Biol Chem 285: 20541-20546, 2010.

32. Li J, Donath S, Li Y, Qin D, Prabhakar BS and Li P: miR-30 regulates mitochondrial fission through targeting p53 and the dynamin-related protein-1 pathway. PLoS Genet 6: e1000795, 2010.

33. Yu F, Deng H, Yao H, Liu Q, Su F and Song E: Mir-30 reduction maintains self-renewal and inhibits apoptosis in breast tumorinitiating cells. Oncogene 29: 4194-4204, 2010. 